\title{
Characteristics and Geographic Dispersion of Syndromic Surveillance Systems in the United States in 2008
}

Sarah B. Lesesne, Lucia Rojas Smith, and David B. Rein

November 2010 


\section{About the Authors}

Sarah B. Lesesne, BS, is a master of science in public health candidate at the University of North Carolina at Chapel Hill in the Department of Health Policy and Management. Previously, she was a research economist in RTI International's Public Health Economics program. She provided research assistance on multiple cost-effectiveness models and worked on the economic evaluation of the BioSense program

Lucia Rojas Smith, DrPH, MPH, is a program evaluator and director of the Health and Social Organizations Research program at RTI. She has more than 20 years' experience leading program evaluations focused on maternal and child health, public health preparedness, and surveillance systems.

David B. Rein, PhD, is a senior research economist in RTI's Public Health Economics program. His work specializes in developing costeffectiveness and disease burden simulation models to directly inform public health decision making.
This publication is part of the RTI Press Research Report series.

RTI International

3040 Cornwallis Road

PO Box 12194

Research Triangle Park, NC

27709-2194 USA

Tel: $\quad+1.919 .541 .6000$

Fax: $\quad+1.919 .541 .5985$

E-mail: rtipress@rti.org

Web site: www.rti.org
RTI Press publication RR-0013-1011

This PDF document was made available from www.rti.org as a public service of RTI International. More information about RTI Press can be found at http://www.rti.org/rtipress.

RTI International is an independent, nonprofit research organization dedicated to improving the human condition by turning knowledge into practice. The RTI Press mission is to disseminate information about RTI research, analytic tools, and technical expertise to a national and international audience. RTI Press publications are peer-reviewed by at least two independent substantive experts and one or more Press editors.

\section{Suggested Citation}

Lesesne, S. B., Rojas Smith, L., and Rein, D. B. (2010). Characteristics and Geographic Dispersion of Syndromic Surveillance Systems in the United States in 2008. RTI Press publication No. RR-0013-1011. Research Triangle Park, NC: RTI Press. Retrieved [date] from http://www.rti.org/rtipress.

\section{Disclaimer}

This report was prepared as an account of work sponsored by an agency of the United States Government. Neither the US Government nor any agency thereof, nor any of their employees, makes any warranty, express or implied, or assumes any legal liability or responsibility for the accuracy, completeness, or usefulness of any information, apparatus, product, or process disclosed, or represents that its use would not infringe privately owned rights. Reference herein to any specific commercial product, process, or service by trade name, trademark, manufacturer, or otherwise does not necessarily constitute or imply its endorsement, recommendation, or favoring by the US Government or any agency thereof. The views and opinions of the authors expressed herein do not necessarily state or reflect those of the US Government or any agency thereof.

C2010 Research Triangle Institute. RTI International is a trade name of Research Triangle Institute.

All rights reserved. Please note that this document is copyrighted and credit must be provided to the authors and source of the document when you quote from it. You must not sell the document or make a profit from reproducing it.

doi:10.3768/rtipress.2010.rr.0013.1011

www.rti.org/rtipress 


\title{
Characteristics and Geographic Dispersion of Syndromic Surveillance Systems in the United States in 2008
}

\author{
Sarah B. Lesesne, Lucia Rojas Smith, \\ and David B. Rein
}

$\begin{array}{lr}\text { Contents } & \\ \text { Introduction } & 2 \\ \text { Methods } & 3 \\ \quad \text { Identification of Programs } & 3 \\ \quad \text { Data Characteristics and } & \\ \quad \text { Aggregation } & 3 \\ \text { Results } & 4 \\ \text { Discussion } & 7 \\ \text { References } & 9 \\ \text { Acknowledgments Inside back cover }\end{array}$

\begin{abstract}
To understand the characteristics, scope, and geographic dispersion of syndromic surveillance systems in the United States in 2008, we reviewed information about their existence and characteristics in each state. We abstracted and compiled data from peer-reviewed literature, public reports, news stories, press releases, and Internet sites; we then analyzed the data, using a systematic instrument. For systems that collected emergency department (ED) information, we estimated the percentage of annual ED visits whose data were reported to the system. In 2008, 39 states and the District of Columbia had syndromic surveillance systems. ED records (65 percent) and hospital admissions records (43 percent) were the most common data sources. Respiratory (57 percent) and gastrointestinal (53 percent) syndromes were the most commonly tracked syndromes. On average, systems using ED records covered 47 percent of all visits, though some states had rates higher than 75 percent. The Northeast, Midwest, and Southeast were more likely to have systems than Western regions. We identified wide variance in the sources of data, the syndromes tracked, and the completeness of syndromic surveillance coverage across states. Although some states have well-established systems that capture diverse data at high levels of coverage, many others have small local systems or none.
\end{abstract}




\section{Introduction}

Syndromic surveillance refers to the use of prediagnostic, health-related data to identify probable disease cases or outbreaks that may require additional response from public health officials. ${ }^{1}$ The goals of syndromic surveillance resemble those of traditional surveillance in that it seeks to create systematic health information and disseminate this information to public health professionals to enhance prevention and control activities. ${ }^{2,3}$ However, several key differences exist between syndromic and traditional surveillance. Syndromic surveillance tracks health care events, rather than diagnoses, and focuses on quickly acquiring and analyzing data as opposed to comprehensively collecting individual-level records. Traditional surveillance places a premium on the accuracy and representativeness of the information it collects, whereas syndromic surveillance prioritizes the breadth of the population used and the timeliness of the information provided.

In the 1990s public health professionals at various state health departments and research institutions conceptualized the first wave of syndromic surveillance systems. ${ }^{4,5}$ They hoped that analysis of the wide-reaching data sources combined in these systems would rapidly help identify emerging health threats, such as pandemic influenza, bioterrorism, and harmful environmental exposures, and would thereby provide early warning and increase the time that public health officials would have to respond. ${ }^{6,7}$ Following the dissemination of anthrax spores in the aftermath of September 11, 2001, the federal government increased funding to support the development and proliferation of syndromic surveillance systems to protect the United States against threats from biological or environmental terrorism. ${ }^{8}$ Since 2001 the number of syndromic surveillance systems has increased dramatically, and the public health community's understanding of possible uses of syndromic surveillance has expanded. Sources estimate that 100 to 200 systems are currently in place throughout state and local public health departments. ${ }^{9,10}$

Although originally innovators intended for syndromic surveillance systems primarily to provide early warning of emerging trends, practitioners have discovered other uses and applications of syndromic data. Syndromic surveillance systems have enhanced public health officials' situational awareness and response capacity. For example, syndromic surveillance systems have been used to track the subsequent health effects of a hurricane, to plan for and distribute medications and vaccinations, to identify the spread of itch mites in an urban community, and to evaluate the populations affected by an extreme heat event. ${ }^{11-13}$ The federal government provides much of the support for the development and expansion of these programs. Private contractors or academic partners have built and maintained other systems, such as the University of Pittsburgh's Real-Time Outbreak Detection System (RODS). ${ }^{14}$ Although syndromic surveillance systems share the overarching goal of early detection of disease outbreaks, they vary widely in system structure, algorithm methodology, and program scope.

Much information has been published on the development of new systems, ${ }^{15-17}$ refinements in analytic methods, ${ }^{18-21}$ and novel uses of active surveillance for identifying trends. ${ }^{12,22}$ Information on the coverage of systems across the United States is more limited, with a few notable exceptions. A 2008 study surveyed state and local health departments and other public health officials involved in syndromic surveillance, providing an aggregated picture of syndromic surveillance nationwide but no information about systems' locations or extent of coverage. ${ }^{9}$ Another recent study used self-reported survey responses from state health departments to examine the presence of syndromic surveillance systems around the country, with a primary focus on system type and software used. ${ }^{23}$ This study also provided limited data on the geographic dispersion of syndromic surveillance systems, offering only information on which states responded to the study's survey requests. It provides no information on whether nonresponding states have syndromic surveillance systems and no information on the level of coverage achieved in each state.

In this study, we present new information on the geographic dispersion of syndromic surveillance systems across the United States and details on the individual syndromes tracked. Additional 
methods for gathering these data may be developed in the future; the information here constitutes a baseline against which to compare future changes to syndromic surveillance. Although we were aware of syndromic surveillance systems scattered throughout the country, we undertook this study to understand how programs were distributed and the extent to which those programs provide surveillance coverage.

\section{Methods}

\section{Identification of Programs}

We identified all city-, county-, and state-level syndromic surveillance activities in each of the 50 US states and the District of Columbia ("states" hereafter) from 1998 to 2008. To do so, we developed a database of existing syndromic surveillance systems and descriptive characteristics, using publicly available peer-reviewed literature, nonacademic reports, news stories, press releases, and other sources available on the Internet. We considered a state to have a system if any locality or jurisdiction within it had a system that met our criteria. We distinguished a syndromic surveillance program from a traditional or sentinel surveillance system by the use of prediagnostic health-related data for early detection of a public health event or disease outbreak.

From February through June 2009, we searched PubMed, Google, and the International Society of Disease Surveillance conference abstracts, ${ }^{24}$ using names of each of the states in conjunction with at least the following search terms: syndromic surveillance, BioSense, and ESSENCE surveillance. BioSense refers to the Centers for Disease Control and Prevention (CDC)-funded, Web-based biosurveillance system that the health care facilities and health departments around the country use for outbreak detection and situational awareness. ${ }^{25}$ ESSENCE refers to the Electronic Surveillance System for the Early Notification of Community-based Epidemics software; several states and military facilities use it to detect public health events by means of hospital emergency department (ED) data. ${ }^{26}$

We included BioSense and ESSENCE as search terms because we expected state or local health departments involved with either BioSense or ESSENCE to be active in syndromic surveillance. On an ad hoc basis, we used other search terms, such as biosurveillance, surveillance, and emergency chief complaint data, among others, to identify systems in states for which our initial search was unable to identify one. However, these additional terms added virtually no information to our original search results.

For a few specific states with little or no publicly available information, we supplemented our search results with short telephone interviews of health department employees. Complying with the Office of Management and Budget's Paperwork Reduction Act limited our ability to survey each state with similar interviews or questionnaires. We excluded information about event-based, temporary, and currently inactive syndromic surveillance activities, such as those implemented specifically for a major sporting event or for wildfires, because such systems will unlikely be reactivated or integrated into a national system. We also excluded systems limited to military institutions or health systems, such as Veterans Administration hospitals and US Department of Defense facilities.

\section{Data Characteristics and Aggregation}

Once the programs were identified, we described each according to the following characteristics:

- the jurisdiction type for the program (city-, county-, or statewide),

- whether the program reported data to a national agency or program,

- the year in which the program started and ended (if applicable), and

- whether the program coordinated with any other syndromic surveillance programs.

We collected information about the types of data used for surveillance and categorized these sources as follows:

- ED, hospital admissions, clinic, or ambulatory center data;

- laboratory data;

- emergency medical services (EMS) or 911 call data;

- over-the-counter drug sales or pharmacy data;

- nurse hotline data; 
- poison control data;

- school absentee data; or

- other.

For those programs using ED data, we collected information that allowed us to calculate the proportion of all local or statewide ED visits covered by the system. Finally, we collected information on whether systems were designed automatically to monitor the following disease syndromes:

- gastrointestinal,

- influenza-like illness,

- respiratory,

- neurological,

- rash,

- fever,

- sepsis, and

- other.

We aggregated local and county data to the state level by combining measures for each system in our database. We assumed that information predating 2008 was still current, and we carried forward data fields, without modification, through 2008, unless specific information indicated that the system was discontinued. We took these measures to ensure that the results would represent the available state-level information on systems from 1998 through 2008.

Using several methods, we created our measure of the proportion of ED visits covered by syndromic surveillance systems in each state. For statewide systems, we used information on statewide ED coverage if such information was available from published sources or from interviews. Some states contained one or more local or county systems that reported the number or proportion of visits covered within the locality but did not present statewide information. In these states, we created a state estimate by combining the proportion of ED visits covered in localities with a system with the proportion covered in areas without a system (assumed to be zero) and then weighting each locality in accordance with its proportion of the state population. Finally, some states contained local- or county-level programs that reported the number of hospitals covered by each system. For these states, we estimated the proportion of ED visit coverage by dividing the number of hospitals participating in the locality by the number of hospitals with EDs in that locality. To accomplish this task, we used the Joint Commission's Quality Check system, which gives information about accredited hospitals nationwide. ${ }^{27}$

We assumed that all hospitals in a locality served approximately the same sized population, and we weighted the proportion of hospitals involved in the system, or systems, by the population of the areas participating. We used a similar weighting method for localities that reported strictly the number of people in their area who were covered by the syndromic surveillance system. We assumed that states with syndromic surveillance systems that did not use ED data and that states for which we could not find evidence of a syndromic surveillance system had no ED coverage.

In winter and spring 2009, we collected, coded, and recorded data for analysis, using Microsoft Excel. Using SAS software, version $9.1,{ }^{28}$ we calculated the proportion of, and the 95 percent confidence interval for, states with each descriptive characteristic. We used a geographic information system to visually display the results. ${ }^{29}$ Sources of data for the syndromic surveillance system were depicted with symbols, and syndrome categories were coded with letters. Color gradients were used to distinguish varying ED coverage rates, while states with either no program or no ED-based program were shown in white.

\section{Results}

We identified 39 states and the District of Columbia with syndromic surveillance systems that were initiated from 1998 to 2008, representing 78 percent of all states (Table 1). ED records, the most common source of data, were collected by 33 states (65 percent). This result compares with

- 22 states (43 percent) that collected hospital or clinic admissions data,

- 14 states (27 percent) that collected pharmacy or over-the-counter drug sales data,

- 10 states (20 percent) that collected school absentee records, 
- 8 states (16 percent) that collected poison control data, and

- 8 states (16 percent) that collected EMS or 911 call data.

Only 6 states ( 12 percent) collected laboratory data, and only 4 ( 8 percent) collected nurse hotline data.
In addition to these commonly cited data sources, 14 states (27 percent) included other data sources as part of their syndromic surveillance activities. Examples of other sources of data included information from wildlife and animal control centers, radiology records, and death certificates. Descriptions of data sources varied by state and by the source of information.

\section{Table 1. Characteristics of US syndromic surveillance activities in 2008}

\begin{tabular}{|c|c|c|c|}
\hline Item & N & Proportion & 95th Cl \\
\hline States with programs & 40 & 0.78 & {$[65.0-87.7 \%]$} \\
\hline States with systems $1-2$ years old & 5 & 0.13 & {$[5.2-27.0 \%]$} \\
\hline States with systems $3-4$ years old & 9 & 0.23 & {$[12.0-38.1 \%]$} \\
\hline States with systems $5-6$ years old & 8 & 0.20 & [10.2-35.4\%] \\
\hline States with systems $7-8$ years old & 14 & 0.35 & {$[21.8-51.0 \%]$} \\
\hline States with systems 9 years old or older & 4 & 0.10 & [3.8-24.0\%] \\
\hline States tracking emergency department data & 33 & 0.65 & {$[50.7-76.6 \%]$} \\
\hline States tracking hospital or clinic admissions & 22 & 0.43 & [30.2-57.0\%] \\
\hline States tracking lab data & 6 & 0.12 & {$[5.3-24.0 \%]$} \\
\hline States tracking emergency medical services or 911 data & 8 & 0.16 & {$[8.0-28.5 \%]$} \\
\hline States tracking over-the-counter or pharmacy data & 14 & 0.27 & [16.9-41.3\%] \\
\hline States tracking nurse hotline data & 4 & 0.08 & [2.9-19.3\%] \\
\hline States tracking poison control & 8 & 0.16 & [8.0-28.5\%] \\
\hline States tracking school absenteeism & 10 & 0.20 & {$[10.8-33.0 \%]$} \\
\hline States tracking other data sources & 14 & 0.27 & {$[16.9-41.3 \%]$} \\
\hline States tracking gastrointestinal syndromes & 27 & 0.53 & [39.6-65.8\%] \\
\hline States tracking influenza-like-illness syndrome & 22 & 0.43 & [30.1-57.2\%] \\
\hline States tracking respiratory syndromes & 29 & 0.57 & [43.5-69.3\%] \\
\hline States tracking neurological syndromes & 20 & 0.39 & {$[26.5-53.6 \%]$} \\
\hline States tracking rash syndromes & 23 & 0.45 & [31.9-59.0\%] \\
\hline States tracking fever syndromes & 20 & 0.39 & {$[26.5-53.6 \%]$} \\
\hline States tracking sepsis syndromes & 11 & 0.22 & [11.7-36.3\%] \\
\hline States tracking other syndromes & 27 & 0.53 & [39.7-65.7\%] \\
\hline States that coordinated with other systems & 11 & 0.22 & {$[12.0-35.6 \%]$} \\
\hline States that coordinated with BioSense & 5 & 0.45 & {$[0.3-77.2 \%]$} \\
\hline
\end{tabular}


For example, discussion of ED systems ranged from the monitoring of "chief complaints of individuals presenting at 10 hospital EDs" 10 to a simple note documenting the initiation of "emergency department (ED)-based syndromic surveillance."30 Similarly, we documented a variety of descriptions of over-the-counter or pharmacy databases used for active surveillance, although the exact source of these data, such as the National Retail Data Monitor or another retail pharmacy database, was often unclear.

States also monitored a wide range of syndromes. Respiratory diseases were the most frequently cited syndrome (tracked by 29 , or 57 percent, of all states), followed by gastrointestinal syndromes (tracked by 27, or 53 percent, of all states). This result compares with 23 (45 percent) states that tracked rash syndromes and 22 (43 percent) that monitored influenza-like-illnesses. Fever syndromes and neurological syndromes followed closely behind, each with 20 (39 percent) states, while only 11 (22 percent) tracked sepsis. Additionally, 27 (53 percent) of states cited monitoring some other syndrome category not listed, including

- hepatitis illness,

- meningitis illness,

- asthma,

- injuries, and

- myocardial infarction.

We were able to calculate the proportion of ED visits covered in 28 of the 33 states that collected ED data (Figure 1). On average, systems in these states captured 47 percent of ED visits, but this rate

Figure 1. Syndromic surveillance in the United States, 2008

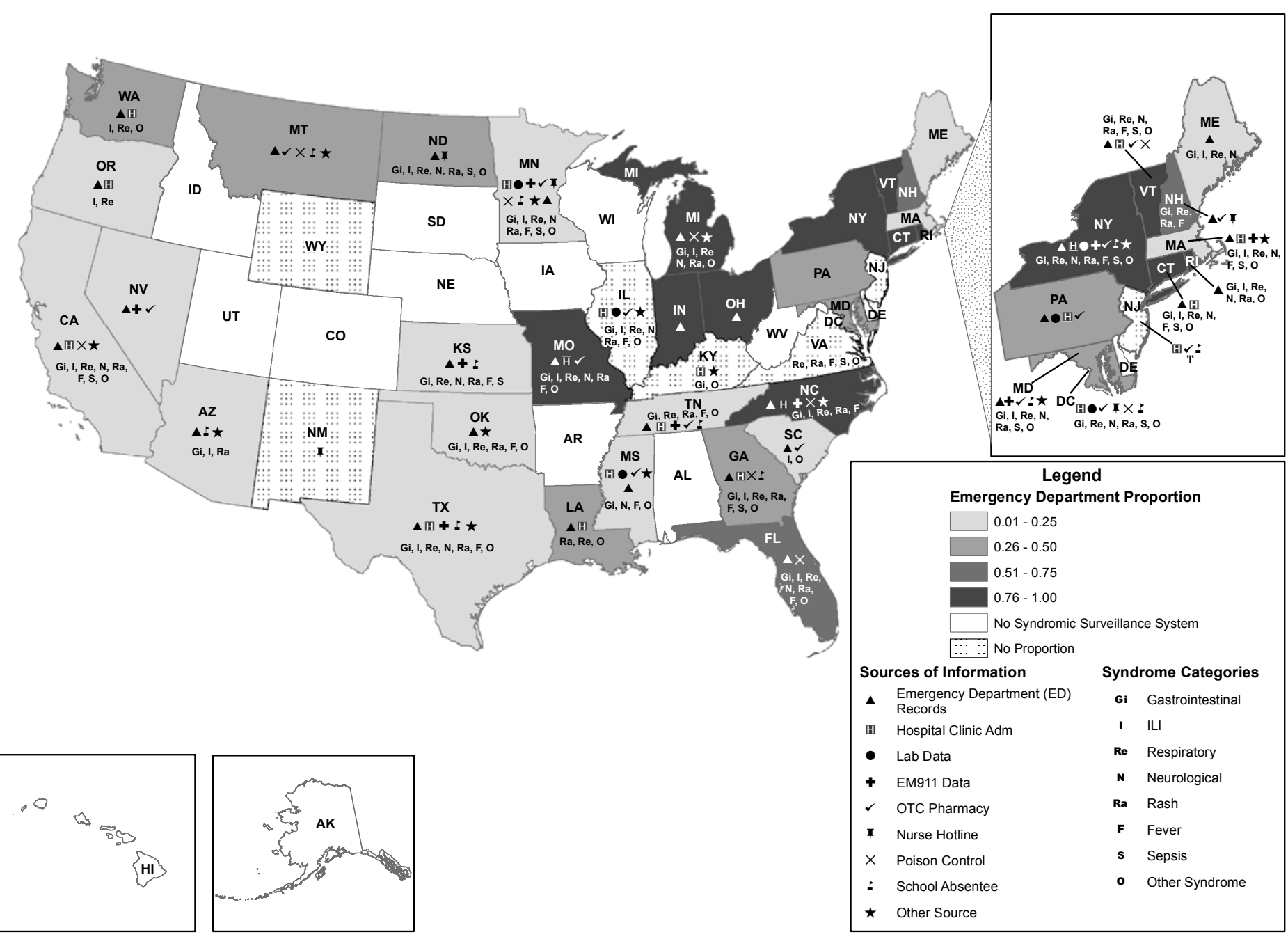


varied widely from a low of 2 percent to a high of 100 percent. For those states in which we could calculate a proportion,

- 10 (36 percent of states) covered 1 percent to 25 percent of visits,

- 7 (25 percent of states) covered 26 percent to 50 percent of visits,

- 2 (7 percent of states) covered 51 percent to 75 percent of visits, and

- 9 (32 percent of states) covered 76 percent to 100 percent of visits.

Of the 40 states with active programs,

- only 5 (13 percent) reported initiating syndromic surveillance in the past 1 to 2 years,

- 9 (23 percent) reported initiating these activities 3 to 4 years ago, and

- 8 (20 percent) reported initiating these activities 5 to 6 years ago.

More than one-third of states (14, or 35 percent) began using syndromic surveillance 7 to 8 years ago, while only 4 (10 percent) began more than 8 years ago. Of the 40 states, 11 (28 percent) reported that they coordinated or shared data with a syndromic surveillance partner. Of these 11 states, 5 reported coordinating activities with the federal CDC BioSense program.

Our data showed some small geographic patterns. Of the 11 states with no syndromic surveillance systems, 7 (Arizona, Iowa, Idaho, Nebraska, South Dakota, Utah, and Wisconsin) were located west of the Mississippi River; 2 (Alaska and Hawaii) were located outside the continental United States. Of the 28 states for which we were able to calculate an ED proportion, half were located in the Midwest or Northeast. The Midwest and Northeast exhibited the highest ED coverage rates, with 93 percent for the Midwest and 54 percent for the Northeast. By contrast, we were able to estimate ED coverage level for only 24 percent of states in the West.

\section{Discussion}

In 2008,40 states ( 80 percent) conducted syndromic surveillance. Although more than two-thirds of these states collected ED records, wide variations in the percentage of visits captured reveals that some areas have far more extensive coverage than others. We were unable to collect data on the coverage level of other types of syndromic surveillance data (for example, school absentee data), but we speculate that similar variations in coverage apply to those systems.

While numerous states had well-developed networks of syndromic surveillance activities, we identified several states with either no system in place, or with systems that covered only limited areas of the state. More than one-third of the states with systems first initiated their systems within the past 7 to 8 years. Potentially, some of these states developed their systems in response to availability of funding for biosurveillance and emergency preparedness after September 11, 2001. These states may be at a higher risk than others of abandoning their systems if federal support for syndromic surveillance meets with future decreases.

Our results are consistent with three other relevant studies on the subject, which lends validity to our findings. In a 2008 study that surveyed state and local health department epidemiologists, Buehler and colleagues reported a total of 40 states with syndromic surveillance activities. ${ }^{9}$ Although this finding strikingly resembles our 39 states and the District of Columbia, we could not determine whether Buehler et al. identified the same states as our study, because they reported only aggregate data. ${ }^{9}$ Buehler et al. also reported that

- 84 percent of states used ED data;

- 49 percent of states collected hospital admissions data, which contrasts with our finding of 57 percent; and

- the Northeast and Midwest had the most syndromic surveillance activity.

A more recent study surveying state public health departments across the country found 33 states with at least one syndromic surveillance activity operating within each state. ${ }^{23}$ This study used a combination 
of published literature and surveys to conduct a comprehensive review of syndromic surveillance systems in the United States. ${ }^{13}$ Although this study identified fewer states with syndromic surveillance activities, the sources of data used by these systems resembled those identified in our study: states most commonly tracked ED records, hospital admissions, and over-the-counter drug sales.

The following factors warrant mention as limitations to our study. First, because our research relied on public records, reports, and information generally available through structured Internet searches, we cannot be certain that we identified all relevant syndromic surveillance systems or all data describing the systems we did identify. This limitation may have affected our results in several ways: syndromic surveillance activities that have not resulted in a published article, announcement, press release, or other source identifiable on the Internet were not captured. Furthermore, data accuracy is likely lessened for states that have published a limited amount of information about their systems or that have not updated Internet descriptions of their systems for several years. Either a primary survey of public health informatics administrators, or a registry of syndromic surveillance systems and their characteristics, would likely yield both richer and more accurate information. This report represents our best attempt to create information in the absence of a survey or registry system.

Second, our aggregation of systems to the state level masks a substantial amount of intrastate variation. For example, California has active programs in Los Angeles County, Monterrey County, Santa Clara County, and San Diego, which cover a substantial proportion of those particular areas. However, other areas of the state have no syndromic surveillance coverage whatsoever. Our New York State data masks that the state operates two independent sets of systems, one managed by the state health department in Albany and another managed by the New York City Department of Health and Mental Hygiene. Additionally, although we report that some of the states lacked syndromic surveillance programs, evidence exists that temporary programs had operated at one time and that some legislatures were considering future programs.

Large federal programs like CDC's BioSense have provided some organization to syndromic surveillance program development in the United States. The large number of independent syndromic surveillance activities, however, makes accurately describing the national picture of syndromic surveillance difficult. Our results do show that the characteristics and coverage of syndromic surveillance programs vary widely across the United States. Although our results present a baseline description of these activities, a centralized registry of local, state-level, and regional syndromic surveillance programs would foster cooperation and partnerships across localities. These partnerships could enhance the utility of individual systems and promote the building of a national system, if such a system is determined to be desirable.

Our study shows that much activity and innovation are occurring locally and regionally, but these systems are rarely coordinated, and substantial areas of the country remain uncovered. Policy makers may want to consider creating programmatic and financial incentives to integrate local systems into regional networks and to integrate regional networks into national systems. 


\section{References}

1. Centers for Disease Control and Prevention. Syndromic surveillance: an applied approach to outbreak detection. 2008 [cited 2009 May 13]; Available from: http://www.cdc.gov/ncphi/disss /nndss/syndromic.htm

2. Buehler J. Surveillance. In: Rothman KJ, Greenland S, editors. Modern epidemiology. 2nd ed. Philadelphia, PA: Lippincott-Raven; 1998.

3. Henning KJ. What is syndromic surveillance? MMWR Morb Mortal Wkly Rep. 2004;53 (Supplement):5-11.

4. Heffernan R, Mostashari F, Das D, Besculides M, Rodriguez C, Greenko J, et al. New York City syndromic surveillance systems. MMWR Morb Mortal Wkly Rep. 2004;53(Supplement):23-7.

5. Mandl KD, Overhage JM, Wagner MM, Lober WB, Sebastiani P, Mostashari F, et al. Implementing syndromic surveillance: a practical guide informed by the early experience. J Am Med Inform Assoc. 2004 Mar-Apr;11(2):141-50.

6. Centers for Disease Control and Prevention. Preventing emerging infectious diseases: a strategy for the 21 st century. Overview of the updated CDC plan. MMWR Morb Mortal Wkly Rep. 1998 Sep 11;47(RR-15):1-14.

7. Centers for Disease Control and Prevention Strategic Planning Workgroup. Biological and chemical terrorism: strategic plan for preparedness and response. Recommendations of the CDC Strategic Planning Workgroup. MMWR Morb Mortal Wkly Rep. 2000 Apr 21; 49(RR-4): 1-14.

8. Centers for Disease Control and Prevention. Update: investigation of anthrax associated with intentional exposure and interim public health guidelines, October 2001. MMWR Morb Mortal Wkly Rep. 2001 Oct 19;50(41):889-93.

9. Buehler J, Sonricker A, Paladini M, Soper P, Mostashari F. Syndromic surveillance practice in the United States: findings from a survey of state, territorial, and selected local health departments. Adv Dis Surveill. 2008;6(3).
10. Kirkwood A, Guenther E, Fleischauer AT, Gunn J, Hutwagner L, Barry MA. Direct cost associated with the development and implementation of a local syndromic surveillance system. J Public Health Manag Pract. 2007 Mar-Apr;13(2):194-9.

11. Cook County Department of Public Health. Annual report 2007. Chicago, IL: Cook County Department of Public Health, Cook County Health and Hospitals System; 2007 [cited 2010 September 23]. Available from: http://www.cookcountypublichealth.org/files/pdf /CCDPH_annrep07.pdf

12. Murray KO, Kilborn C, DesVignes-Kendrick M, Koers E, Page V, Selwyn BJ, et al. Emerging disease syndromic surveillance for Hurricane Katrina evacuees seeking shelter in Houston's Astrodome and Reliant Park Complex. Public Health Rep. 2009 May-Jun;124(3):364-71.

13. Yan P, Chen H, Zeng D. Syndromic surveillance systems: public health and biodefense. Annual Review of Information Science and Technology (ARIST). 2008;42.

14. Espino JU, Wagner M, Szczepaniak C, Tsui FC, $\mathrm{Su} \mathrm{H}$, Olszewski R, et al. Removing a barrier to computer-based outbreak and disease surveillance-the RODS Open Source Project. MMWR Morb Mortal Wkly Rep. 2004 September 24;53 Suppl:32-9.

15. Hadler JL, Siniscalchi A, Dembek Z. Hospital admissions syndromic surveillance-Connecticut, October 2001-June 2004. MMWR Morb Mortal Wkly Rep. 2005 Aug 26;54 Suppl:169-73.

16. Heffernan R, Mostashari F, Das D, Karpati A, Kulldorff M, Weiss D. Syndromic surveillance in public health practice, New York City. Emerg Infect Dis. 2004 May;10(5):858-64.

17. Miller B, Kassenborg H, Dunsmuir W, Griffith J, Hadidi M, Nordin JD, et al. Syndromic surveillance for influenzalike illness in ambulatory care network. Emerg Infect Dis. 2004;10(10): 1806-11. 
18. Buckeridge DL, Burkom H, Moore A, Pavlin J, Cutchis P, Hogan W. Evaluation of syndromic surveillance systems-design of an epidemic simulation model. MMWR Morb Mortal Wkly Rep. 2004 Sep 24;53 Suppl:137-43.

19. Burkom HS, Elbert Y, Feldman A, Lin J. Role of data aggregation in biosurveillance detection strategies with applications from ESSENCE. MMWR Morb Mortal Wkly Rep. 2004 Sep 24;53 Suppl:67-73.

20. Burkom HS, Murphy S, Coberly J, Hurt-Mullen K. Public health monitoring tools for multiple data streams. MMWR Morb Mortal Wkly Rep. 2005 Aug 26;54 Suppl:55-62.

21. Cooper G, Dowling J, Levander J, Sutovsky P. A Bayesian algorithm for detecting CDC Category A outbreak diseases from emergency department chief complaints. Adv Dis Surveill. 2007;2:45.

22. Das D, Metzger K, Heffernan R, Balter S, Weiss $\mathrm{D}$, Mostashari F. Monitoring over-the-counter medication sales for early detection of disease outbreaks-New York City. MMWR Morb Mortal Wkly Rep. 2005;54(Suppl):41-6.

23. Uscher-Pines L, Farrell CL, Cattani J, Hsieh YH, Moskal MD, Babin SM, et al. A survey of usage protocols of syndromic surveillance systems by state public health departments in the United States. J Public Health Manag Pract. 2009 Sep-Oct;15(5):432-8.
24. International Society for Disease Surveillance. Advances in disease surveillance. 2006-2008 [cited 2009 May 31]; Available from: http://www.isdsjournal.org

25. Centers for Disease Control and Prevention. About BioSense. 2009 [cited 2010 September 23]; Available from: http://www.cdc.gov/BioSense/

26. Lombardo JS, Burkom H, Pavlin J. ESSENCE II and the framework for evaluating syndromic surveillance systems. MMWR Morb Mortal Wkly Rep. 2004;53 Suppl:159-65.

27. The Joint Commission. Quality check. 2009 [cited 2009 May 31]; Available from: http://www.qualitycheck.org

28. SAS/STAT (Version 9.1) [computer software]. Cary, NC: SAS; 2004.

29. ArcGIS (Version 9.3) [computer software]. Redlands, CA: ESRI; 1995-2010.

30. Miller S, Fallon K, Anderson L. New Hampshire emergency department syndromic surveillance system. J Urban Health. 2003;80(Suppl 1):I118-9. 


\section{Acknowledgments}

We would like to thank and acknowledge Peter J. Leese, MSPH, for his early contributions to the research for this work. We also wish to thank Craig Hales, MD, MPH, Jerome Tokars, MD, MPH, and Taha Kass-Hout, MD, MS, for their contributions and support. The Centers for Disease Control and Prevention provided financial support through cooperative agreement U38 HK000009. 
RTI International is an independent, nonprofit research organization dedicated to improving the human condition by turning knowledge into practice. RTI offers innovative research and technical solutions to governments and businesses worldwide in the areas of health and pharmaceuticals, education and training, surveys and statistics, advanced technology, international development, economic and social policy, energy and the environment, and laboratory and chemistry services.

The RTI Press complements traditional publication outlets by providing another way for RTI researchers to disseminate the knowledge they generate. This PDF document is offered as a public service of RTI International. More information about RTI Press can be found at www.rti.org/rtipress. 